\title{
Where methanol masers spring
}

\author{
Karl J. E. Torstensson ${ }^{1,2}$, Huib Jan van Langevelde ${ }^{2,1}$ \\ and Stephen Bourke ${ }^{3}$ \\ ${ }^{1}$ Leiden Observatory \\ P.O. Box 9513, 2300 RA Leiden, The Netherlands \\ email: kalle@strw.leidenuniv.nl \\ ${ }^{2}$ Joint Institute for VLBI in Europe \\ Postbus 2, 7990 AA Dwingeloo, The Netherlands \\ email: langevelde@jive.nl \\ ${ }^{3}$ National University of Ireland, Galway \\ Department of Information Technology, National University of Ireland, Galway, Ireland \\ email: stephen@it.nuigalway.ie
}

\begin{abstract}
We are carrying out a program to observe the $6.7 \mathrm{GHz}$ methanol maser emission in high-mass star-forming regions using large FOV $\left(\sim 2^{\prime}\right)$, astrometric, VLBI data. Here we report on the first results of the inner few arc seconds in Cepheus A East. We find a maser filament extending over $\sim 1.7$ " (1200 AU), straddling the waist of Cep A HW2. The region in which the $\mathrm{CH}_{3} \mathrm{OH}$ masers are found contains several YSO's and it is not clear whether the $\mathrm{CH}_{3} \mathrm{OH}$ masers are associated with several different objects or rather the larger scale surrounding environment.
\end{abstract}

Keywords. masers, stars: formation, stars: individual (Cepheus A)

\section{Introduction}

At a distance of $725 \mathrm{pc}$, Cepheus A East is a nearby complex high-mass star-forming region. At large scale several outflows are seen in many molecular species (e.g. Codella et al. 2005). In the centimetre regime the continuum emission is dominated by several compact sources and the bipolar jets associated with Cep A HW2. It is not yet clear how many of the compact sources are associated with individual YSO's. Also, in the region close to HW2 several YSO's have been reported in a number of tracers (Martín-Pintado et al. 2005, Curiel et al. 2002, Patel et al. 2005, Brogan et al. 2007). In this poster we report on recent results of the $6.7 \mathrm{GHz}$ methanol masers in the region of Cep A HW2.

\section{Observations}

The observations were carried out in November 2004 with 8 telescopes of the EVN. Cep A was observed for $\sim 2 \mathrm{~h}$ including calibrators. The observations were split into two $1 \mathrm{~h}$ blocks, separated by a few hours to improve the uv-coverage. The experiment was setup at a centre frequency of $6668.5142 \mathrm{MHz}$ (rest frequency) with $2 \mathrm{MHz}$ bandwidth and 1024 channels, resulting in a velocity resolution of $\sim 0.1 \mathrm{~km} \mathrm{~s}^{-1}$. Both RCP and LCP were recorded. The data was correlated at JIVE with an integration time of $0.25 \mathrm{~s}$.

All calibration and imaging was done in AIPS. Amplitude and bandpass calibration was done on 3C345 and DA193, observed at the beginning and end of each block. For phase calibration and initial self-calibration J2302+6405 was observed every three minutes. The brightest maser feature was then used to determine the absolute position and as a source for further self-calibration, resulting in an rms of $\sim 3.5 \mathrm{mJy} \mathrm{beam}^{-1}$ in the emission free channels. 


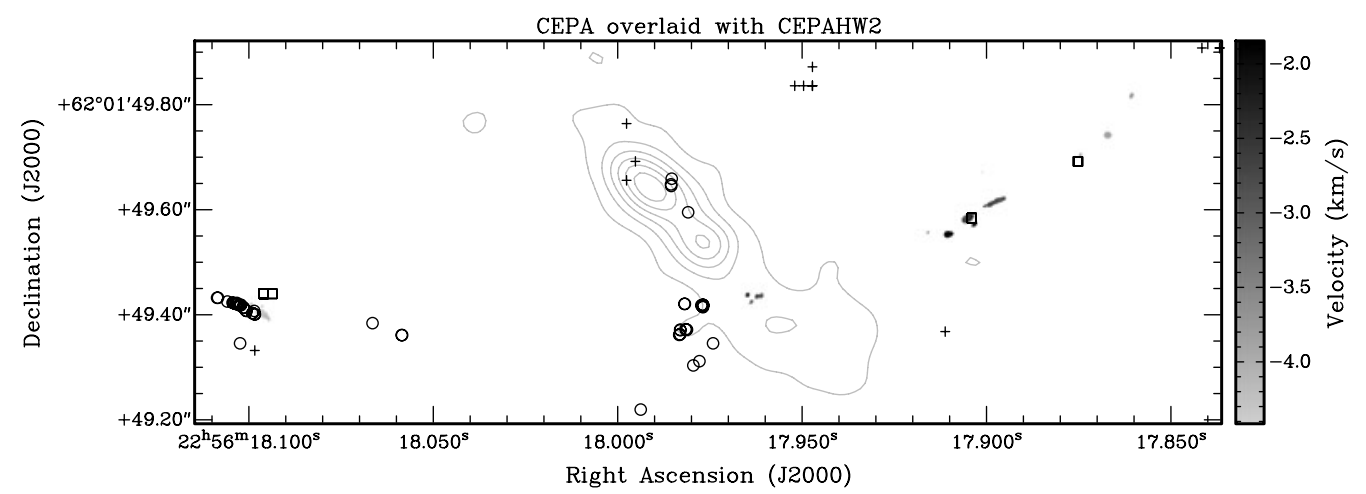

Figure 1. The $6.7 \mathrm{GHz} \mathrm{CH}_{3} \mathrm{OH}$ masers (grey scale) overlaid on $\mathrm{K}$ band continuum (contours) (Torrelles et al. 1998). Also shown are the $12.2 \mathrm{GHz} \mathrm{CH}_{3} \mathrm{OH}$ masers (boxes) (Minier et al. 2001), water masers (circles) (Vlemmings et al. 2006) and OH masers (pluses) (Bartkiewicz et al. 2005).

\section{Discussion}

In Cep $\mathrm{A}$ we find the $\mathrm{CH}_{3} \mathrm{OH}$ maser emission to be arising from an extended $\sim 1.7$ " ( 1200 AU) narrow filamentary structure, which seems to suggest a single underlying structure. Across the whole structure there is a small velocity gradient but without obvious signs of rotation. The maser positions to the west correspond well to the positions of the $12.2 \mathrm{GHz} \mathrm{CH}_{3} \mathrm{OH}$ masers measured by Minier et al. (2001). To the east the positional agreement is poorer, this could simply be an effect of observations at different epochs. Overall, the velocity of the $6.7 \mathrm{GHz}$ masers agrees well with that of the $12.2 \mathrm{GHz}$ masers.

In all regions except one the $\mathrm{CH}_{3} \mathrm{OH}$ masers are spatially offset from other maser species in the region (water and $\mathrm{OH}$ ). Interestingly, the methanol masers to the east are spatially coincident with the water masers observed by Vlemmings et al. (2006), although they show a velocity offset of $\sim 10 \mathrm{~km} \mathrm{~s}^{-1}$. The weak maser emission arising from the very waist of HW2 is very interesting as it may point toward the very object responsible for the outflows/jets. It is however not coincident with the $7 \mathrm{~mm}$ continuum object identified by Curiel et al. (2006), but a position further to the SW in the jet.

In fact, none of the proposed YSO's in this region (Martín-Pintado et al. 2005, Curiel et al. 2002, Patel et al. 2005, Brogan et al. 2007) show a direct, small scale, association with any $\mathrm{CH}_{3} \mathrm{OH}$ maser. If the masers are arising in a single structure it seems difficult to attribute their origin to a single object. It is remarkable that a structure encompassing several young stars has such a small velocity gradient.

\section{References}

Bartkiewicz, A., Szymczak, M., Cohen, R. J., et al. 2005, MNRAS 361, 623

Brogan, C. L., Chandler, C. J., Hunter, T. R., et al. 2007, arXiv:astro-ph/0703626v1

Codella, C., Bachiller, R., Benedettini, M., et al. 2005, MNRAS 361, 244

Curiel, S., Trinidad, M. A., Cantó, J., et al. 2002, ApJL 564, L35

Curiel, S., Ho, P. T. P., Patel, N. A., et al. 2006, ApJ 638, 878

Martín-Pintado, J., Jiménez-Serra, I., Rodríguez-Franco, A., et al. 2005, ApJL 628, L61

Minier, V., Conway, J. E. \& Booth, R. S. 2001, A\&A 369, 278

Patel, N. A., Curiel, S., Sridharan, T. K., et al. 2005, Nature 437, 109

Torrelles, J. M., Gómez, J. F., Garay, G., et al. 1998, ApJ 509, 262

Vlemmings, W. H. T., Diamond, P. J., van Langevelde, H. J., et al. 2006, A\& A 448, 597 